\title{
PluriTAV: desarrollo de las competencias plurilingües mediante el uso de la traducción audiovisual ${ }^{1}$
}

\author{
Anna Marzàa , Gloria Torralba Miralles ${ }^{\mathrm{b}}$, Beatriz Cerezo Merchán ${ }^{\mathrm{c}}$ y Juan José Martínez \\ Sierrad $^{\mathrm{d}}$
}

a'Universitat Jaume I, amarza@edu.uji.es, 'bniversitat Jaume I, gtorralb@edu.uji.es, 'Universitat de València, beatriz.cerezo@uv.es y ${ }^{\mathrm{d}}$ Universitat de València, juan.j.martinez@uv.es.

\begin{abstract}
This piece of work introduces the PluriTAV (Audiovisual Translation as a Tool for the Development of Multilingual Competences in the Classroom) research project. This project is staked on one of the most recent approaches of foreign language teaching, the multilingual approach, in which not only the language being studied (or taught) but also the linguistic repertoire of the classroom are taken into account. The project, focused on the teaching and perfecting of language, is based on the said approach and draws on a field related to foreign language teaching, audiovisual translation, so that through it, one can learn a foreign language, perfect mother tongue abilities and develop multilingual abilities. The fundamental objective of this project consists in the creation of a free web platform (PluriTAV) that allows the exploration and exploitation of the methodological possibilities that the implementation of the multilingual approach and the tools of different audiovisual translation modes can offer in the teaching of a foreign language and in the perfecting of mother tongues in the university environment. The hypothesis is that the proposed approach will allow improvement in foreign language acquisition, perfection of first languages and promotion of multilingual skills.
\end{abstract}

Keywords: didactics, foreign/mother tongue, audiovisual translation, multilingual approach, competences, multimedia resources

\section{Resumen}

En este trabajo se presenta el proyecto de investigación PluriTAV. La traducción audiovisual como herramienta para el desarrollo de competencias plurilingües en el aula. Se trata de un proyecto que apuesta por uno de los enfoques más recientes de enseñanza de lenguas extranjeras (LE), el multilingüe, en el que se tienen en cuenta no solo la lengua que se estudia, sino también el repertorio lingüístico del aula. El proyecto, centrado en la didáctica

\footnotetext{
${ }^{1}$ Esta investigación se enmarca en el proyecto de investigación PluriTAV. La traducción audiovisual como herramienta para el desarrollo de competencias plurilingües en el aula (ref. FFI2016-74853-P, 2017-2019), financiado por la Agencia Estatal de Investigación (AEI) y el Fondo Europeo de Desarrollo Regional (FEDER).
} 
y el perfeccionamiento de lenguas, se basa en dicho enfoque y recurre a un campo que tiene su espacio dentro de la didáctica de LE, la traducción audiovisual, para, a partir de ella, aprender una LE, perfeccionar competencias de las lenguas propias y desarrollar competencias plurilingües. El objetivo fundamental consiste en la creación de una plataforma web libre y gratuita (PluriTAV) que permita explorar y explotar las posibilidades metodológicas que la aplicación del enfoque multilingüe y de herramientas basadas en diferentes modalidades de traducción audiovisual pueden ofrecer a la enseñanza de lenguas extranjeras y al perfeccionamiento de las maternas en el ámbito universitario. La hipótesis es que el enfoque propuesto permitirá mejorar la adquisición de LE, perfeccionar las maternas y potenciar las competencias plurilingües.

Palabras clave: didáctica, lengua extranjera/materna, traducción audiovisual, enfoque multilingüe, competencias, recursos multimedia

\section{Introducción}

Vivimos en una sociedad cada vez más globalizada y multilingüe, algo que, unido a la importancia internacional del inglés, la profusión de las situaciones multilingües en entornos laborales y académicos y el mejorable nivel de lenguas extranjeras (LE), propicia un claro reto de mejora de las competencias plurilingües. La didáctica de lenguas debe seguir ofreciendo nuevas perspectivas interdisciplinarias para la adquisición de LE y para la mejora de las maternas, y el proyecto que aquí se presenta pretende buscar nuevos enfoques desde campos afines y nuevas herramientas para ampliar y mejorar los conocimientos lingüisticos.

Este proyecto apuesta por uno de los enfoques más recientes de enseñanza de LE, el multilingüe, en el que se tienen en cuenta no solo la lengua que se estudia, sino también el repertorio lingüístico del aula, para el diseño de los objetivos, las tareas y la evaluación. El proyecto, centrado en la didáctica y el perfeccionamiento de lenguas, se basa en dicho enfoque y recurre a un campo que tiene su espacio dentro de la didáctica de LE, la traducción audiovisual (TAV), para, a partir de ella, aprender una LE, perfeccionar competencias de las lenguas propias y desarrollar competencias plurilingües.

El objetivo fundamental consiste en la creación de una plataforma web libre y gratuita (PluriTAV) que permita explorar y explotar las posibilidades metodológicas que la aplicación del enfoque multilingüe y de herramientas basadas en diferentes modalidades de TAV (como doblaje, subtitulación, voice over, audiodescripción o SPS) pueden ofrecer, inicialmente, a la enseñanza de la lengua inglesa y al perfeccionamiento del catalán y el español en el ámbito universitario. La hipótesis es que el enfoque propuesto permitirá mejorar la adquisición de LE, perfeccionar las lenguas maternas y potenciar las competencias plurilingües.

Como principal resultado figurará PluriTAV, una plataforma de didáctica de la lengua inglesa, española y catalana desde un enfoque multilingüe, destinada a docentes y estudiantes y que tendrá, como rasgo distintivo, la aplicación de las herramientas propias de diversas 
modalidades de TAV dirigidas a la enseñanza de lenguas, con el Marco Común Europeo de Referencia para las Lenguas (MCER) y el Marco de Referencia para los Enfoques Plurales de las Lenguas y de las Culturas (MAREP) como guías.

Como se verá, a diferencia de otros proyectos que también han conectado la TAV y la didáctica de lenguas, este se centra en la práctica totalidad de modalidades de TAV, y adopta el enfoque multilingüe para evaluar su impacto en todas las lenguas implicadas en el intercambio lingǘstico y, en especial, para explorar la influencia que la transferencia interlingüística propia de la tarea traductora puede ejercer en las competencias plurilingües de los alumnos. Asimismo, se dará relevancia a la figura del docente.

\section{Objetivos e hipótesis}

Como ya se ha mencionado, el objetivo fundamental de este proyecto consiste en la creación de una plataforma web de acceso libre en la que los docentes de lenguas podrán encontrar fundamentación teórica y recursos didácticos para la aplicación de diferentes modalidades de TAV en la enseñanza de lenguas, con una perspectiva multilingüe. Todo esto partiendo del convencimiento de que el enfoque propuesto permitirá mejorar la adquisición de ciertas competencias lingüísticas específicas y desarrollar la competencia multilingüe por parte del alumnado, con la ventaja añadida de que los resultados obtenidos podrán ser fácilmente extrapolables y aplicables a otras lenguas y a otros niveles de formación. Entre otras cuestiones se pretende, a partir de un repaso inicial a las diferentes teorías de enseñanza de lenguas extranjeras y del papel que la traducción ha tenido en ellas, definir propuestas de uso didáctico de la TAV para el desarrollo de competencias específicas y llevar a cabo un proyecto eminentemente aplicado, con experimentación directa en el aula, de modo que los resultados obtenidos no sean fruto de abstracciones o suposiciones, sino de la previa comprobación empírica de las líneas de trabajo propuestas. Todo ello en un contexto social en el que existe una clara apuesta por el aprendizaje de idiomas y por la movilidad en todos los niveles educativos en el ámbito europeo, contexto en el que este proyecto busca encontrar nuevas vías para llevar a cabo dicha pretensión.

El actual contexto lingüístico, cultural, comercial, político, social y económico, con una destacada movilidad de ciudadanos que gestionan, además del inglés, una elevada diversidad lingüística, requiere nuevos planteamientos que contribuyan a aumentar el nivel de lenguas que los profesionales precisen, sea cual sea el fin que le den a esas segundas (o terceras) lenguas (familiar, profesional, escolar, social, comunidades concretas...). La didáctica de lenguas extranjeras, como ya se ha dicho, está obligada a seguir buscando fórmulas que contribuyan a avanzar en el objetivo general de formar a futuros profesionales cada vez más capacitados lingüísticamente.

A la hora de reflexionar sobre la viabilidad didáctica y pedagógica del proyecto es importante plantearse también cuál será la direccionalidad traductora que se requerirá en la implementación de los materiales. Hoy en día se reconoce la utilidad de la traducción inversa $(\mathrm{L} 1 \rightarrow \mathrm{LE})$ como herramienta para el aprendizaje de lenguas (Yuste Frías, 2005: 155). El hecho de traducir a una lengua extranjera suele conllevar una mayor dificultad que hacerlo hacia la 
materna, aunque esto también puede resultar un aliciente que fomente el esfuerzo. Lertola (2012: 200), por su parte, cree que la traducción directa es una actividad que resulta más útil para detectar los problemas y errores más comunes entre el alumnado, mientras que la traducción inversa resulta más adecuada desde un punto de vista pedagógico. La vasta profusión de material audiovisual y las facilidades de acceso a este que ofrecen las nuevas tecnologías hacen posible contar con recursos tanto en L1 como en LE, y esto permite una infinidad de usos dependiendo del nivel del alumnado y de los objetivos de la tarea. Además, cabe destacar que el enfoque adoptado por los citados autores, tanto para el diseño de la investigación como para el análisis de resultados, no es multilingüe y, por tanto, faltan estudios que evalúen de forma global los beneficios de una traducción directa o inversa en el aprendizaje de las dos lenguas utilizadas en el intercambio lingüístico.

Así, partimos de la hipótesis general de que será posible encontrar en el campo de la TAV conceptos, planteamientos y herramientas (desde actividades hasta software) que nos permitan analizar, delimitar y describir un recurso didáctico poco explorado hasta la fecha y que puede contribuir a la mejora de las capacidades comunicativas en tres ámbitos: la mejora de la lengua extranjera (inglés, en este proyecto), el perfeccionamiento de algunas competencias de las lenguas maternas o de instrucción general (español o catalán en el contexto en el que se lleva a cabo este proyecto) y el desarrollo de determinadas competencias plurilingües que se activan al trabajar simultáneamente en dos lenguas del repertorio.

\section{Antecedentes y estado actual}

\subsection{Consideraciones generales}

En una sociedad cada vez más globalizada, los intercambios de información entre distintos países en sus correspondientes lenguas son continuos, si bien la lengua inglesa sigue imperando como lingua franca en numerosos ámbitos ${ }^{2}$. Sin embargo, la tendencia en el mundo empresarial y académico es la de ir incluyendo el uso progresivo de más lenguas, lo cual refleja la realidad de unas sociedades cada vez más multilingües. De hecho, autores como Janssens y Steyaert (2014) destacan que en los mercados internacionales coexisten diversas lenguas y que más que de una lingua franca, es preciso hablar de una multilingua franca ${ }^{3}$. Esto encaja con la visión del proyecto, puesto que el inglés será la lengua común en todos o la mayoría de los ejercicios (también porque es la que forma parte del currículo español), pero a través del uso de esta lengua y de las propias (español y catalán), nos centraremos en las competencias que necesitarán desarrollar los alumnos para un futuro decididamente

\footnotetext{
${ }^{2}$ Por ejemplo, el inglés es el idioma oficial en más de 50 países, y está presente en ámbitos tan variados como la Unión Europea, Naciones Unidas o el Comité Olímpico Internacional, siendo además la lengua utilizada con más frecuencia en los intercambios comerciales entre países. Es, además, el idioma oficial de comunicación aeronáutica y marítima.

${ }^{3}$ Es igualmente relevante mencionar el estudio ELAN.cat: http://noticias.universia.es/vidauniversitaria/noticia/2010/04/08/654445/multilingismo-instrumento-negocio.html.
} 
multilingüe en el que puede que no haya una única lingua franca, sino que los fenómenos de alternancia de lenguas sean la norma en la empresa, como ya lo son en la sociedad.

Pese a esta realidad lingüística, la sociedad española sigue presentando como una asignatura pendiente la mejora del dominio de idiomas. Tomando el inglés como ejemplo, según datos facilitados en 2015 por la EF Índice de Nivel de Inglés, la primera y más completa clasificación de las destrezas del idioma inglés en adultos alrededor del mundo, España aparece en un puesto intermedio (23) en el ámbito internacional. Ahora bien, en el marco europeo nuestro país cae hasta las últimas posiciones. De manera similar, según la Encuesta sobre la Participación de la Población Adulta elaborada por el Instituto Nacional de Estadística (INE) de 2011, el 47,24\% de la población no sabe ninguna lengua además de la materna, un $34,78 \%$ habla una lengua extranjera, un $13,45 \%$ dos y solo un $4,54 \%$ tres o más. Esto nos conduce de nuevo a un escenario multilingüe en el que la lengua inglesa figura como primera lengua extranjera (LE) ${ }^{4}$ en cuanto a uso, conocimiento y presencia en el currículo educativo.

Por tanto, si triangulamos 1) el actual entorno globalizado (medios de comunicación, formación, negocios...); 2) la importancia de la lengua inglesa en el ámbito internacional y el empuje de las situaciones multilingües en entornos laborales; y 3) el mejorable porcentaje de conocimientos de lenguas extranjeras, la conclusión palmaria que obtenemos es que nuestra sociedad tiene como un claro reto el fomento o mejora de las competencias plurilingües, con objeto de no perder las diferentes oportunidades, fundamentalmente de mercado y profesionales, que el ámbito internacional le brinda.

A partir de esta consideración, y como se sugería, la didáctica de lenguas tiene la obligación de ofrecer de manera insistente nuevas perspectivas y metodologías que permitan seguir dando pasos en la dirección señalada. Entendemos que la interdisciplinariedad es un valor que dicha didáctica ha de aprovechar. Por tanto, merece la pena seguir evolucionando y dirigir nuestra mirada a otros campos afines en busca de nuevos enfoques y herramientas que nos permitan enseñar lenguas y desarrollar competencias multilingües de una manera cada vez más eficiente y completa. Uno de dichos campos afines, como se apuntaba, lo constituye la traducción, más concretamente, la TAV. El interés por este ámbito y sus posibilidades de aplicación y explotación por parte de la didáctica de lenguas extranjeras se encuentra en una fase relativamente inicial, aunque ha dejado ya entrever un sinfín de posibilidades. En esta línea se enmarca el proyecto que aquí se presenta, aunque con un enfoque claramente diferenciador: el aprovechamiento del contacto entre lenguas que permite de forma natural la traducción, para explotar el desarrollo de las competencias multilingües de los alumnos, que se añadiría a la mejora de las competencias específicas en cada lengua, y la generación de una plataforma que posibilite la explotación de dicho contacto.

\footnotetext{
${ }^{4}$ A menudo, cuando se utiliza el concepto segunda lengua (L2), se hace referencia al estudio de cualquier lengua a parte de la materna (L1). Sin embargo, en contextos bilingües, tales como aquel en el que se desarrolla este proyecto, es necesario distinguir entre segunda lengua y lengua extranjera (LE). Así pues, utilizamos la nomenclatura L1 o L2 para apelar al español o al catalán, dependiendo de cuál sea la lengua materna del alumno, y con LE nos referiremos a la lengua inglesa.
} 
Como antes se apuntaba, la principal finalidad de este proyecto consiste en generar una plataforma que permita evaluar el uso de la traducción como herramienta para el aprendizaje de lenguas desde una perspectiva multilingüe, tomando como base la aplicación de enfoques y herramientas (así como software) relacionados con las diferentes modalidades de TAV. De manera paralela, se busca ahondar en la investigación y aplicación del enfoque multilingüe, e implementar una serie de actividades en línea que vinculen las competencias de la adquisición lingüística a dichas modalidades de TAV, así como utilizar software libre para TAV que facilite este aprendizaje. Para ello, y dada la naturaleza interdisciplinaria del proyecto, se hace necesario un repaso de los antecedentes en este campo.

Respecto al proyecto propiamente dicho, no podemos dejar de mencionar las investigaciones cuya temática está relacionada con la de la que aquí se presenta. Los dos antecedentes más claros son los proyectos europeos LeViS y ClipFlair. El primero, ejecutado en 2008, partió de la premisa, aquí compartida, de que cada vez es más evidente para profesores e investigadores de lenguas extranjeras que no existe un único método garantizado de enseñanza y que es preciso combinar en clase varios recursos técnicos y metodológicos. Pretendía, fundamentalmente, generar herramientas y métodos para contrarrestar la pasividad que se puede producir al trabajar con material audiovisual; es decir, fomentar el papel activo del estudiante mediante el aprendizaje activo, haciendo uso de los multimedia de manera productiva como núcleo de la actividad (principalmente, centrada en la subtitulación) y no como un mero elemento complementario (http://levis.cti.gr/). Por su parte, y en una línea similar, ClipFlair, ejecutado en 2015, tenía como punto de partida el hecho de que los docentes que usan recursos audiovisuales se encuentran con la dificultad de encontrar tareas activas que hagan participar a los estudiantes y disuadan la observación pasiva. Por ello, se postulaba la idea de que una forma de trabajar con material audiovisual de manera productiva y motivadora es pidiendo a los estudiantes que doblen o subtitulen un vídeo (http://clipflair.net/).

El presente proyecto, si bien comparte diferentes características con los dos citados en el párrafo anterior, posee una serie de elementos distintivos. Por un lado, no se centra en una única modalidad de TAV (como el subtitulado, en el caso de LeViS), sino en la práctica totalidad de modalidades con mayor presencia en nuestro país (atendiendo a su pertinencia en nuestro contexto social más cercano e incluyendo, por ejemplo, la audiodescripción o las voces superpuestas). Por otro, y de manera esencial, a diferencia de lo que se presenta en LeViS y Clipflair, en este proyecto trasladamos la realidad bilingüe de la tarea que llevarán a cabo los alumnos al diseño de la investigación y adoptamos el enfoque multilingüe propuesto por Cenoz y Gorter (2011), según el cual la investigación no debería centrarse en una única lengua, sino en el repertorio lingüístico del alumnado y en las relaciones entre las lenguas de dicho repertorio. Este enfoque da respuesta a la exigencia del MCER de transformar el objetivo de la enseñanza de lenguas para lograr desarrollar un único repertorio lingüístico y ofrecer al alumnado la posibilidad de ampliar su competencia plurilingüe. Así pues, y teniendo en cuenta el contexto en el que se desarrolla, en este proyecto evaluaremos empíricamente el impacto que la TAV tiene en las dos lenguas entre las que se producirá el intercambio lingüístico (inglés y español o catalán) y, además, exploraremos la influencia que la transferencia interlingüística propia de la tarea traductora puede ejercer en las competencias plurilingües de los alumnos, unas competencias que no van ligadas a ninguna lengua concreta, sino a las

(cc) EY-NC-ND 2018, Universitat Politècnica de València

Congreso IN-RED (2018) 
relaciones entre estas. Finalmente, mientras que los dos proyectos citados se centraban en el estudiante, el nuestro tiene también en consideración la figura del docente, puesto que no se trata únicamente de generar un catálogo de actividades, sino también de (re)elaborar unos fundamentos investigadores, teóricos y metodológicos que permitan a dicho docente hacer uso de la plataforma para, por ejemplo, crear sus propias actividades y utilizar los instrumentos de evaluación de desarrollo de competencias elaborados en el proyecto para medir su efectividad.

\subsection{Principales teorías de la didáctica de lenguas y su relación con la traducción}

A lo largo del siglo XX hemos sido testigos de la aplicación de un variado número de métodos y enfoques para el aprendizaje de idiomas. La traducción ha jugado distintos papeles a lo largo de la evolución de la didáctica de lenguas. En la actualidad todavía son muchos los que la relacionan con el método Gramática-Traducción, el cual se impuso en el campo de la enseñanza de lenguas hasta mediados del siglo XX, y consistía, básicamente, en enseñar una lengua combinando la explicación de normas gramaticales con la traducción directa e inversa de frases. La artificialidad de esta metodología y la poca atención que le dedicaba a aspectos como las destrezas orales, conllevaron que se viera desplazada por otras que abogaban por la inmersión lingüística en el aula y el monolingüismo en la lengua estudiada, realidad que ocasionó la total ausencia de traducción, salvo como ejercicio literario para estudiantes de nivel avanzado (Zaro, 1999: 533).

Hubo que esperar hasta principios de la década de los ochenta del pasado siglo para que, gracias a metodologías como los enfoques comunicativos (en los que la comunicación es el objetivo final en el aprendizaje de idiomas) y los enfoques basados en tareas (que presentan situaciones familiares a los alumnos a través de tareas, para que así aprendan a utilizar el lenguaje adecuado en situaciones reales concretas), la traducción volviera a utilizarse como recurso didáctico y se valorara su carácter de actividad comunicativa auténtica.

El final del siglo XX trajo consigo algunas críticas a la rigidez de los enfoques y métodos de enseñanza de lenguas que se estaban adoptando. Muchos enseñantes se encontraban ante situaciones en las que, a pesar de haber apostado por un enfoque o método concretos, las circunstancias del aula les obligaban a adaptarlo o a combinarlo con otros. Esta búsqueda de eclecticismo favoreció el nacimiento del Postmétodo, una nueva postura que reclamaba una revisión de la pedagogía en términos de estrategias de aula, objetivos curriculares, materiales docentes y evaluación (Kumaravadivelu, 2001), algo que, a su vez, contribuyó a que se volviera a introducir la actividad de traducción en la enseñanza de lenguas extranjeras. En este sentido, autores como Cook recuerdan que si lo que se buscan son tareas reales en las que prime el significado y la comunicación sea el objetivo último, la traducción cumple todos los requisitos, dado que es "a real-world activity outside the classroom". El autor añade que "It is outcome-orientated: a successful translation is one that works. It promotes a focus on form as an offshoot of a communicative need, rather than as an end in itself" (2010: 30).

La perspectiva multilingüe ha acabado de situar a la traducción en un lugar destacado en la enseñanza de lenguas, desde que Manyak (2004) observó que la traducción desde su lengua materna promovía la adquisición del inglés como lengua extranjera, el desarrollo de la lectura 
y la escritura bilingüe y la afirmación de la propia identidad entre los alumnos. Autores como Cummins (2014) o Malakoff y Hakuta (1991) también defienden el uso de la traducción en el aula no solo para promover la adquisición de la lengua extranjera, sino también para desarrollar o afianzar la lengua materna. Con este proyecto, como antes se apuntaba, se pretende contribuir a esta línea de investigación con una metodología experimental, para determinar qué competencias mejoran gracias al uso de la traducción.

En nuestro caso, todos los elementos positivos que incluye la traducción como herramienta para el aprendizaje de lenguas extranjeras son extrapolables a la especialidad de la TAV, aunque la naturaleza polisemiótica del texto audiovisual hace necesario tener en consideración aspectos que no están presentes en la traducción general.

\subsection{El MCER y el MAREP}

Nuestro proyecto se desarrolla dentro del actual contexto europeo, en lo que a los niveles de lenguas se refiere. Por tanto, no podemos obviar el marco común del que se ha dotado. Según explica el Centro Virtual Cervantes, el MCER proporciona una base común para la elaboración de programas de lenguas, orientaciones curriculares, exámenes, manuales... en toda Europa. Describe de forma integradora lo que tienen que aprender a hacer los estudiantes de lenguas con el fin de utilizar una lengua para comunicarse, así como los conocimientos y destrezas (saber hacer) que tienen que desarrollar para poder actuar de manera eficaz. La descripción también comprende el contexto cultural donde se sitúa la lengua. El MCER define, asimismo, niveles de dominio de la lengua que permiten comprobar el progreso de los alumnos en cada fase del aprendizaje y a lo largo de su vida.

Por último, el MCER coloca a la traducción entre las destrezas (saber hacer) que un hablante competente debe poseer, al incluir la mediación entre las actividades que activan la competencia lingüística comunicativa de dicho hablante. Según este documento, el objetivo de la enseñanza de lenguas para los ciudadanos de la Unión Europea no es la competencia comunicativa en una lengua concreta, sino una competencia plurilingüe y pluricultural que permita no solo la comunicación, sino también la intermediación entre diferentes lenguas y culturas (es decir, la comunicación intercultural).

Desde esta perspectiva, la finalidad de la educación en una lengua queda profundamente modificada. Ya no se contempla como el simple logro del dominio de una o dos (o incluso tres) lenguas, cada una considerada de forma aislada, con el hablante nativo ideal como modelo fundamental. Por el contrario, el objetivo es el desarrollo de un repertorio lingüístico en el que tengan lugar todas las capacidades lingüísticas. Esto supone, naturalmente, que las lenguas que se ofrecen en las instituciones educativas tienen que diversificarse y que a los alumnos debe dárseles la posibilidad de desarrollar una competencia plurilingüe, lo cual exige ciertas modificaciones metodológicas que aboguen por una mayor integración en la didáctica de lenguas y por un enfoque más multilingüe en el diseño de las tareas y la evaluación, ambos aspectos considerados en este proyecto.

Aun así, en el MCER las competencias se encuentran divididas por lenguas y no se especifica con detalle las características de dicha competencia plurilingüe. Por esta razón, desde el Centro 
Europeo de Lenguas Modernas (ECML, por sus siglas en inglés), perteneciente al Consejo de Europa, se ha propuesto un marco de referencia complementario al MCER y específico para las competencias plurilingües: el MAREP.

Dicho marco describe una serie de competencias globales propias de los hablantes plurilingües, válidas para toda lengua y toda cultura y también para las relaciones entre lenguas y entre culturas. Asimismo, propone una serie de recursos como elementos que pueden ejercitarse en clase y contribuir a desarrollar dichas competencias. Este marco nos servirá como referencia para la elaboración de las actividades y los instrumentos de evaluación referidos a las competencias plurilingües, entendiendo por competencia "un savoir-agir complexe résultant de l'intégration, de la mobilisation et de l'agencement d'un ensemble de capacités et d'habiletés (pouvant être d'ordre cognitif, affectif, psychomoteur ou social) et de connaissances (connaissances déclaratives) utilisées efficacement, dans des situations ayant un caractère commun” (Lasnier, 2000: 32).

\subsection{La traducción audiovisual}

Podemos definir la TAV como una variedad de traducción que se ocupa de los textos audiovisuales, los cuales se caracterizan, fundamentalmente, por transmitir información a través de dos canales simultáneos y complementarios (el acústico y el visual) y por presentar una combinación de varios códigos de significación (lingüístico, paralingüístico, visual, etc.) cuyos signos interactúan y construyen el entramado semántico del texto. Muestra una serie de características propias que la definen frente a la traducción escrita y a la interpretación y que tienen que ver, principalmente, con los condicionantes o restricciones que presenta y las estrategias que requiere.

El doblaje y la subtitulación son las principales modalidades de TAV, al menos cuantitativamente. Las voces superpuestas están también presentes en nuestro contexto más inmediato (por ejemplo, documentales). Ahora bien, existen otras modalidades a las que deseamos prestar especial atención por su relevancia en nuestro contexto social y que merecen ser visibilizadas con el fin de evitar la sensación de que la actividad traductora audiovisual se reduce a la práctica del doblaje y de la subtitulación.

La subtitulación para sordos difiere de la convencional en que incluye información referente a la banda sonora (música, efectos especiales...) y también suprasegmental, como la entonación de los actores a la hora de hablar, a la cual las personas con deficiencias auditivas no tienen acceso. Incluye, además, otras características como un uso de léxico y de estructuras sintácticas más sencillas de lo habitual. Por su parte, la audiodescripción para ciegos, que implica una traducción de naturaleza semiótica, tiene como principal objetivo convertir en palabras la información visual de un texto audiovisual. En esta modalidad se intercalan comentarios sobre los aspectos más significativos de la imagen en los huecos entre los diálogos de producciones como pueden ser una película, una obra de teatro o una ópera.

A estas modalidades, más habituales en los medios, cabe añadir otras como el comentario libre, el rehablado, el sobretitulado o la traducción para el teatro. Por último, merece la pena nombrar otras manifestaciones de TAV que escapan al paraguas oficial de modalidades, y que 
son, básicamente, variantes de prácticas como el doblaje o la subtitulación llevadas a cabo no por profesionales. Se trata de los fandubs y los fansubs, doblajes y subtitulaciones hechas por amateurs y de gran popularidad entre el público más joven.

\subsection{La TAV y la didáctica de lenguas}

Nuestro proyecto se basa en la utilización de la traducción pedagógica como instrumento didáctico al servicio de la adquisición de competencias plurilingües. Se han constatado diferencias entre los niveles de competencia lingüística de los distintos países europeos, si se agrupan según la tendencia predominante en términos de TAV. En un informe relativamente reciente de la Comisión Europea (2012) sobre el aprendizaje de lenguas extranjeras en Europa, el porcentaje de encuestados capaces de hablar al menos una lengua diferente a la suya era, en el caso de España (país doblador), de un 48\%, lejos de los porcentajes de países subtituladores como Holanda (94\%), Suecia (91\%) o Dinamarca (89\%)

La mayoría de estudios sobre TAV y enseñanza de lenguas se han centrado en la subtitulación, quizá porque se ha considerado como la modalidad más adecuada de la TAV para el aprendizaje de una lengua extranjera. No obstante, como explica Chaume (2004: 147), las aplicaciones de otras modalidades de TAV y, en especial, del doblaje, constituyen un campo aún prácticamente virgen. El experimento de Danan (2010) y el trabajo de von Flotow (2009) apuntan a la posibilidad del uso del doblaje como instrumento totalmente válido para la enseñanza de lenguas. Con el enfoque adoptado en este proyecto, en el cual también se analiza el efecto de la TAV en la mejora de las competencias en las lenguas curriculares (español y catalán), se abren las posibilidades de aprovechamiento de las distintas combinaciones lingüísticas que ofrecen las diversas modalidades de TAV.

Este es, pues, un aspecto innovador del presente proyecto, puesto que pretende explorar las posibilidades de otras modalidades de TAV hasta la fecha ignoradas o apenas consideradas en el contexto de la didáctica de lenguas extranjeras, como pueden ser las voces superpuestas, la audiodescripción o el comentario libre (con un gran potencial para la redacción creativa), por ejemplo.

Por otro lado, como también señala Talaván (2013: 34), la gran multitud de medios audiovisuales de los que disponemos en la actualidad ayudan a crear un entorno de enseñanzaaprendizaje de una lengua extranjera activo, multisensorial y participativo, en el que los materiales audiovisuales se pueden utilizar de un modo productivo, eficiente y motivador. Por ello, resulta crucial analizar el papel que juegan las nuevas tecnologías de la información y la comunicación (TIC) y el vídeo (el medio audiovisual por excelencia) en la enseñanzaaprendizaje de una lengua extranjera. Si al recurso semiótico del producto audiovisual se le

\footnotetext{
${ }^{5}$ Países cuyo nivel de inglés es, sin embargo, más bajo que el nivel acreditado en España. Por tanto, quizá no se deba establecer necesariamente una relación entre subtitulación y alto nivel de inglés, puesto que hay países subtituladores cuyo nivel de inglés es bajo. Sin duda, influyen otros factores, como la inversión e investigación en la enseñanza de lenguas. De ahí la necesidad de proyectos como este.
} 
añade el elemento de la autenticidad (del que disponemos con gran facilidad en entornos multimedia de internet), se obtienen contextos de aprendizaje bastante realistas. A pesar de que el vídeo es uno de los ejemplos más representativos de este tipo de materiales auténticos necesarios, desgraciadamente se ha utilizado de un modo limitado durante años (Baltova, 1994; Canning-Wilson, 2000; Mayer, 2002; o Talaván, 2007). En la actualidad, la presencia de los medios audiovisuales es altamente notable, si bien no es tan frecuente su uso para llevar a cabo tareas activas (y no pasivas, como sí se hace con el contenido multimedia ${ }^{6}$ ). Por otro lado, tampoco es habitual usar los audiovisuales para llevar a cabo situaciones reales de comunicación, como se plantea en este proyecto. Los alumnos ejercerán el rol de fansubbers y fandubbers, puesto que llevarán a cabo todo el proceso: desde el borrador de traducción hasta la sincronización y ajuste de voces (y locución de diálogos) o subtítulos, dando como resultado un producto audiovisual acabado.

Una idea influyente que también justifica el uso de las TIC y del vídeo y que ha dominado el pensamiento occidental de las últimas décadas es el concepto de las inteligencias múltiples, que explica que la inteligencia no es una facultad mental monolítica, sino que tiene más de un nivel. Tanto las TIC como el vídeo son recursos idóneos para activar distintos tipos de inteligencia (más allá de la puramente lingüística) de modo simultáneo, por lo que un mayor número de aprendices se puede beneficiar plenamente de su utilización.

Para finalizar este apartado, no podemos dejar de mencionar la importancia que ha adquirido la investigación en enseñanza de lenguas a través de la TAV desde un punto de vista activo, especialmente de la subtitulación. Tal y como se ha comentado, en la última década hemos asistido a la realización de experimentos que han involucrado a los sujetos en la práctica de la traducción y la subtitulación. Así, en sus tesis, Talaván (2009), Bravo (2008) y Lertola (2013) recurrieron a esta modalidad para investigar su grado de relevancia en la mejora de conocimientos relacionados con la comprensión oral, las expresiones idiomáticas y el vocabulario, respectivamente. A estos trabajos, llevados a cabo con estudiantes de lengua extranjera, hay que sumar el de Torralba (2016), que también se centra en la adquisición de léxico a través de la subtitulación activa, pero en este caso con estudiantes del grado de Maestro como sujetos.

Conscientes de la reciente profusión de publicaciones que, de una manera dispersa, ahondan en el campo de la adquisición de lenguas, otra de las aportaciones de este proyecto será la sistematización del estudio de las posibilidades de la TAV en la enseñanza de lenguas, con un enfoque holístico que aborde todas las posibilidades de manera conjunta, cohesionada y completa. En todo caso, en la fase de revisión del estado de la cuestión, además de la de otros estudios publicados por los propios miembros del equipo, es precisa la lectura de trabajos como, por citar algunos, los de 1) doblaje en el aprendizaje de lenguas (AL): Kumai (1996),

\footnotetext{
${ }^{6}$ Como podemos leer en la web de Clipflair, "Using audiovisual material in the foreign language classroom is a common resource for teachers since it introduces variety, provides exposure to nonverbal cultural elements and, most importantly, presents linguistic and cultural aspects of communication in their context. However, teachers using this resource face the difficulty of finding active tasks that would engage learners and discourage passive viewing" (http://clipflair.net/).
} 
Burston (2005), Wagener (2006), Chiu (2012), Navarrete (2013), Martínez Sierra (2014) o Talaván y Ávila-Cabrera (2015); 2) subtitulación en el AL: Williams y Thorne (2000), Vermeulen (2003), Sokoli (2006), Wagener (2006), Bravo (2008), Incalcaterra (2009), Borghetti (2011), Sokoli, Zabalbeascoa y Fountana (2011), Martínez Sierra (2014), Incalcaterra y Lertola (2011 y 2014) o Lertola (2012); o 3) audiodescripción en el AL: Clouet (2005), Martínez Martínez (2012) o Ibáñez Moreno y Vermeulen (2013a y 2013b).

\section{Resultados futuros}

Como antes se avanzaba, entre los resultados palpables del proyecto figurará una plataforma virtual interdisciplinaria, única en su género, de didáctica de lenguas en contextos multilingües basada en la TAV y dirigida al desarrollo de las competencias plurilingües con el MCER y el MAREP como guías. Este sitio web, denominado PluriTAV, será de acceso libre y gratuito y contendrá, entre otros, recursos didácticos (incluyendo actividades y software para el trabajo guiado ${ }^{7}$ ), y estará ideado como plataforma de referencia para profesores y de práctica para estudiantes. PluriTAV (http://citrans.uv.es/pluritav/) incluirá contenidos multimedia de naturaleza didáctica sobre la enseñanza de lenguas desde una perspectiva multilingüe basada en la aplicación de la TAV. Esta plataforma, creada en las tres lenguas propias del contexto de este proyecto (inglés, español y catalán), estará estructurada en distintas secciones que incluirán, entre otros, una explicación de los fundamentos teóricos en los que se engloba la investigación y un apartado de bibliografía (ya disponible), la presentación de los resultados de la misma (como los instrumentos de evaluación), la aplicación de dichos resultados a la actividad docente (catálogo de actividades), el calendario de congresos (incluido el que se celebrará en la Universidad de Valencia, cuya página web se incluirá en este sitio), seminarios y talleres pensados para la difusión de los resultados, software para la traducción y el aprendizaje de lenguas, galería de vídeos clasificados por modalidades de traducción, por competencias específicas y por dificultades, manuales de uso en formato audiovisual y un foro abierto pensado para el intercambio de ideas, además de información sobre el programa que ha subvencionado la investigación y sobre los miembros de los equipos.

Dicho producto supondrá el siguiente beneficio: una mejor comprensión de las posibilidades de los enfoques multilingües y un enriquecimiento de los recursos didácticos de la enseñanza de lenguas, siempre dentro del contexto del MCER, que proporcionará un abanico de novedosas opciones metodológicas para la labor del personal docente.

\footnotetext{
${ }^{7}$ Fruto de la Declaración de la Sorbona (1998) y de la Declaración de Bolonia (1999), Europa comenzó su andadura hacia la creación de un Espacio Europeo de la Educación Superior (EEES). La creación del EEES es ya una realidad, y las universidades europeas se centran ahora en su consolidación. Una de las principales apuestas de este espacio educativo europeo es el cambio de paradigma del proceso de enseñanza-aprendizaje: pasamos de un sistema conductista a un sistema cognitivo-constructivista del conocimiento, en el que el estudiante es el protagonista de su propio proceso de aprendizaje y el profesor actúa como guía o facilitador.
} 
PluriTAV se encuentra en el inicio de su segundo año de vigencia, periodo durante el cual se va a llevar a cabo el diseño y aplicación del experimento pretendido, por lo que aún no dispone de conclusiones que poder aportar.

\section{Referencias}

Baltova, I. (1994). "Impact of video on the comprehension skills of core French students" en Canadian Modern Language Review, 50, 3, p. 506-531.

BorghetTI, C. (2011). "Intercultural Learning through Subtitling: The Cultural Studies Approach” en Incalcaterra, L., Biscio, M., Ní Mhainnín, M. Á. Audiovisual Translation Subtitles and Subtitling. Theory and Practice. Berna: Peter Lang. 111-137.

BRAVO, C. (2008). Putting the reader in the picture: Screen translation and foreign-language learning. Tesis doctoral. Tarragona: Universitat Rovira i Virgili.

BURSTON, J. (2005). "Video dubbing projects in the foreign language curriculum” en CALICO Journal, 23, p. 79-92.

CANNING-Wilson, C. (2000). "Practical Aspects of Using Video in the Foreign Language Classroom” en The Internet TESL Journal, 6, 11.

CENOz, J., GORTER, D. (2011). "Focus on multilingualism: A study of trilingual writing” en Modern Language Journal, 95, p. 356-369.

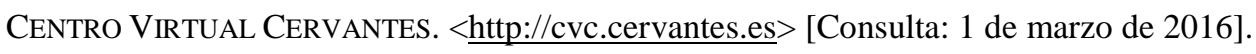

Chaume, F. (2004). Cine y traducción. Madrid: Cátedra.

CHIU, Y. H. (2012). “Can film dubbing projects facilitate EFL learners’ acquisition of English pronunciation?” en British Journal of Educational Technology, 43, 1, p. 24-27.

CLOUET, R. (2005). "Estrategia y propuestas para promover y practicar la escritura creative en una clase de inglés para traductores". En Actas del IX Simposio Internacional de la Sociedad Española de Didáctica de la Lengua y la Literatura. 319-326.

СоoK, G. (2010). Translation in Language Teaching. Oxford: Oxford University Press.

Cummins, J. (2014). "Rethinking pedagogical assumptions in Canadian French immersion programs" en Journal of Immersion and Content-Based Language Education, 2, 1, 3-22.

DANAN, M. (2010). "Dubbing projects for the language learner: a framework for integrating audiovisual translation into task-based instruction” en Computer Assisted Language Learning, 23, 5, p. 441-456.

FLOTOW, L. VON (2009). "Frenching the feature film twice: Or le synchronien au débat" en Díaz Cintas, J. New Trends in Audiovisual Translation. Bristol: Multilingual Matters. 8399.

IBÁÑEZ Moreno, A., Vermeulen, A. (2013a). "La audiodescripción como técnica aplicada a la enseñanza y aprendizaje de lenguas para promover el desarrollo integrado de 
competencias” en Orozco, R. New Directions on Hispanic Linguistics. Baton Rouge: Cambridge Scholars Publishing. 258-287.

--- (2013b). “Audio Description as a tool to improve lexical and phraseological competence in Foreign Language Learning” en Floros, G., Tsigari, D. Translation in Language Teaching and Assessment. Newcastle: Cambridge Scholars Publishing. 41-64.

INCALCATERRA, L. (2009). "Inter-semiotic Translation in Foreign Language Acquisition: The Case of Subtitles” en Witte, A., Harden, T., Ramos de Oliveira Harden, A. Translation in Second Language Learning and Teaching. Berna: Peter Lang. 227-244.

INCALCATERRA, L., LERTOLA, J. (2011). "Learn through subtitling: Subtitling as an aid to language learning” en Incalcaterra, L., Biscio, M., Ní Mhainnín, M. Á. Audiovisual Translation Subtitles and Subtitling. Theory and Practice. Berna: Peter Lang. 243-263.

--- (2014). “Audiovisual translation in second language acquisition. Integrating subtitling in the foreign-language curriculum” en The Interpreter and Translator Trainer, 8, 1, p. 70-83.

JAnssens, M., SteyaerT, C. (2014). "Re-considering language within a cosmopolitan understanding: Toward a multilingual franca approach in international business studies” en Journal of International Business Studies, 45, p. 623-639.

KUMAI, W. (1996). “Karaoke movies: Dubbing movies for pronunciation” en The Language Teacher Online, 20, 9.

Kumaravadivelu, B. (2001). “Toward a Postmethod Pedagogy” en TESOL Quartely, 35, 4, p. 537-560.

LASNIER, F. (2000). Réussir la formation par compétences. Montréal: Guérin.

LertolA, J. (2012). "The effect of subtitling task on vocabulary learning” en Pym, A., Orrego-Carmona, D. Translation research projects 4. Tarragona: Intercultural Studies Group. 61-70.

--- (2013). Subtitling New Media: Audiovisual Translation and Second Language Acquisition. Tesis doctoral. Galway: National University of Ireland.

MALAKOFF, M., HAKUTA, K. (1991). "Translation skill and metalinguistic awareness in bilinguals” en Language Processing in Bilingual Children, p. 141-166.

MANYAK, P. C. (2004). “'What did she say?' Translation in a primary-grade English immersion class” en Multicultural Perspectives, 6, p. 12-18

MARTínEZ MARTínEZ, S. (2012). "La audiodescripción (AD) como herramienta didáctica: Adquisición de la competencia léxica” en Cruces, S., del Pozo, M., Luna, A., Álvarez, A. Traducir en la Frontera. Granada: Atrio. 87-102.

MARTínez Sierra, J. J. (2014). “The Use of Audiovisual Translation Software in Second Language Teaching” en Journal of Foreign Language Teaching and Translation Studies, 3, 1, p. 74-85.

MAYER, R. E. (2001). Multimedia learning. Nueva York: Cambridge University Press.

(cc) EY-NC-ND 2018, Universitat Politècnica de València

Congreso IN-RED (2018) 
NAVARRETE, M. (2013). "El doblaje como herramienta de aprendizaje en el aula de español y desde el entorno de CLIPFLAIR” en MarcoELE, 16.

SOKOLI, S. (2006). "Learning via Subtitling (LvS). A tool for the creation of foreign language learning activities based on film subtitling”, ponencia presentada en Multidimensional translation: Audiovisual translation scenarios, Copenhague.

Sokoli, S., Zabalbeascoa, P., Fountana, M. (2011). "Subtitling Activities for Foreign Language Learning: What Learners and Teachers Think” en Incalcaterra, L., Biscio, M., Ní Mhainnín, M. Á. Audiovisual Translation Subtitles and Subtitling. Theory and Practice. Berna: Peter Lang. 219-242.

TALAVÁN, N. (2009). Aplicaciones de la traducción audiovisual para mejorar la comprensión oral del inglés. Tesis doctoral. Madrid: Universidad Nacional de Educación a Distancia.

--- (2007). "Learning Vocabulary through Authentic Video and Subtitles" en TESOL-SPAIN Newsletter, 31, p. 5-8.

--- (2013). La subtitulación en el aprendizaje de lenguas extranjeras. Barcelona: Octaedro.

Talaván, N., Ávila-Cabrera, J. (2015). "First insights into the Combination of Dubbing and Subtitling as L2 didactic tools" en Gambier, Y., Caimi, A., Mariotti, C. Subtitles and Language Learning. Berna: Peter Lang. 149-172.

TORRALBA, G. (2016). L'aprenentatge de llengües a través de la traduccio audiovisual: la subtitulació com a eina per a l'adquisició de lèxic en llengua estrangera. Tesis doctoral. Castellón: Universitat Jaume I.

Vermeulen, A. (2003). "La traducción audiovisual en la enseñanza de idiomas”. En Actas del Segundo Congreso Internacional de Español para Fines Específicos. 159-168.

WAGENER, D. (2006). "Promoting independent learning skills using video on digital language laboratories” en Computer Assisted Language Learning, 19, 4-5, pp. 279-286.

WiLliams, H., THORNE, D. (2000). "The value of teletext subtitling as a medium for language learning” en System, 28, 2, p. 217-228.

YUSTE FRÍAS, J. (2005). "Didáctica de la traducción inversa español-francés: el fin justifica los medios” en Yuste Frías, J., Álvarez Lugrís, A. Estudios sobre traducción, teoría, didáctica, profesión. Vigo: Universidad de Vigo. 147-170.

ZARO, J. J. (1999). "La traducción, estrategia y objeto de aprendizaje” en Salaberri, R., Sagrario, M. Lingüística aplicada a la enseñanza de lenguas extranjeras. Almería: Universidad de Almería. 531-555. 